\title{
Agglomeration of ultra-fine particles from flue gas in coal-fired power plant using polymeric flocculants
}

\author{
Yi Zhang ${ }^{1, a}$, Kunqi Li $i^{1, b}$, Haoran Chen ${ }^{2, c}$, Yuanfeng $\operatorname{Pan}^{3, d^{*}}$ \\ Huining Xiao ${ }^{1, \mathrm{e}}$,Hua Guo ${ }^{2, \mathrm{f}}$
}
${ }^{1}$ School of Environment Science and Engineering, North China Electric Power University, Baoding 071003, China;
${ }^{2}$ Beijing Key Laboratory of power generation system function materials, Guodian New Energy Technology Research Institute, Beijing 102209, China;
${ }^{3}$ School of Chemistry and Chemical Engineering, Guangxi University, Nanning 530004, China
azhangyi_hd@sina.com, ${ }^{b}$ likunqihg@sina.com, ${ }^{c}$ chenhaoran@cgdc.com.cn, ${ }^{\mathrm{d}}$ panyf@gxu.edu.cn, ehuiningxiao@hotmail.com, ${ }^{\mathrm{f}}$ guohua@cgdc.com.cn

Keywords:polyacrylamides; coal-fired power plant; ultra-fine particles; agglomeration; turbidity Abstract The flocculation or agglomeration of ultra-fine particles from flue gas in coal-fired power plant induced by various polymeric flocculants, polyacrylamides (PAM) in particular, was studied using jar tests. Various influencing factors were investigated, including different type, molecular weight and charge density of polyacrylamides. Results showed that cationic polyacrylamides (CPAM) performed better than anionic and nonionic PAM; whereas cationic PAMwith a high molecular weight and charge density exhibited best flocculation performance. Specifically, when CPAMwas added at $3 \mathrm{ppm}$ to $0.2 \% \mathrm{w} / \mathrm{w}$ of ultra-fine suspension, the turbidity of the suspension was successfully lowered by $99.1 \%$; and the average size of agglomeratedultra-fine particles was increased up to 80 times.

\section{INTRODUCTION}

Ultra-fine particles with diameter less than $2.5 \mu \mathrm{m}(\mathrm{PM} 2.5)$ has attracted much attention due to strong environmental concerns on air pollution or haze problems in recent years. Because of small particle size and low sedimentation rate,ultra-fine particles are enriched with hazardous substances. As the energy structure of China,the ultra-fine particles emitted from coal-fired power contribute to the air pollution significantly ${ }^{[1]}$. The conventional precipitator such as electrostatic precipitator(ESP)and bag filters have considerable collection efficiency of the particles greater than $10 \mu \mathrm{m}$ in diameter ${ }^{[2]}$. However, the collection efficiency for ultra-fine particles is poor. Techniques using agglomeration methods to control emitting of ultra-fine particles from coal-fired power plant include electric agglomeration ${ }^{[3]}$, acoustic agglomeration ${ }^{[4]}$ and chemical agglomeration. The ultrafine particles after agglomeration can be easily captured by conventional precipitator.

Chemical agglomerationoften uses polymeric flocculants to spray into flue gas to collide with ultra-fine particles, thus inducing the agglomeration of ultra-fine particles. As a water-soluble linear polymeric flocculants, polyacrylamides have strong flocculation performance with colloidal particles in aqueoussystem, increasing the size of the particles.

In this work the flocculation performance of polyacrylamides acting on ultra-fine particles with jar test was studied as a foundation for the ultra-fine particles agglomeration in flue gas of coal-fired power plant. Various influencing factors were investigated, including different type, molecular weight and charge density of polyacrylamides.

\section{EXPERIMENTAL}

\section{Materials}

Ultra-fine particles were obtained by grinding the fly ash from coal-fired power plant, with particle size ranging from $0.46 \mu \mathrm{m}$ to $11.2 \mu \mathrm{m}$ (av. Diameter $2.57 \mu \mathrm{m}$ ) and negative charge density at $6.5 \mu \mathrm{eq} / \mathrm{g}$. Each ultra-fine particles sample was freshly diluted to a $0.2 \% \mathrm{w} / \mathrm{w}$ solution with distilled water, which turbidity of the supernatant was ranged from 2000 to 3500 NTU. Different type, 
molecular weight and charge density of polyacrylamides were freshly diluted to $0.1 \% \mathrm{w} / \mathrm{w}$ solution with distilled water. Characteristics of each polyacrylamide are given in Table 1.

Table 1Polymer product description

\begin{tabular}{|c|c|c|c|}
\hline Polymer type & Name & MW (million) & Supplier \\
\hline Anionic polyacrylamide & AP-1000 & 10 & $\begin{array}{l}\text { Sinopharm Chemical } \\
\text { Reagent CO.,Ltd. }\end{array}$ \\
\hline $\begin{array}{c}\text { Non-ionic } \\
\text { polyacrylamide }\end{array}$ & NP-800 & 8 & $\begin{array}{l}\text { Shanghai Hengli Water } \\
\text { Treatment MaterialsLtd }\end{array}$ \\
\hline Cationic polyacrylamide & CP-9020 & 8 & Same as above \\
\hline Cationic polyacrylamide & Percol 164 & $\sim 8$ & BASF SE Chemicals \\
\hline Cationic polyacrylamide & Percol 63 & $\sim 4$ (highcationic) & BASF SE Chemicals \\
\hline Cationic polyacrylamide & Percol 292 & $\sim 4$ (low cationic) & BASF SE Chemicals \\
\hline
\end{tabular}

\section{Flocculation jar test}

In the flocculation experiment, different dosages of polyacrylamides solution $(0.1 \% \mathrm{wt})$ were added into $100 \mathrm{~mL}$ of ultra-fine particles suspension $(0.2 \% \mathrm{wt})$. The supernatant of suspension was extracted from $0.5 \mathrm{~cm}$ underneath the suspension surface after stirring at $200 \mathrm{r} / \mathrm{min}$ for $3 \mathrm{~min}$ and settling for $10 \mathrm{~min}$. The turbidity of suspension supernatant was measured using a $2100 \mathrm{~N}$ Spectrophotometric turbidity meter from $\mathrm{HACH}$.

To compare the degree of ultra-fine particle agglomeration induced by variouspolymers, the turbidity removal rate was measured, which is defined as the difference between the turbidity of suspension supernatantbefore and after agglomeration; and was calculated using the equation (1).

$$
\tau_{r}=\frac{\tau_{i}-\tau_{f}}{\tau_{i}} \times 100 \%
$$

Where ${ }^{\tau_{r}}$ is turbidity removal rate; ${ }^{\tau_{f}}$ denotes final suspension supernatant turbidity; and $\tau_{i}$ stands for unflocculated suspension supernatant turbidity.

\section{Measurement of particle size}

The particle size and distribution of ultra-fine particles was measured before and after agglomeration of flocculation using a Mastersizer 3000 laser particle size analyzer (Malvern Ins.).

\section{RESULTS AND DISCUSSION}

\section{Effect of polyacrylamide type}

Figure1 shows the results of flocculation efficiency of anionic,non-ionic and cationic polyacrylamidewith similar molecular weight to ultra-fine particles. It appears that theturbidity removal rate of ultra-fine particles suspension wasincreased rapidly as the dosage of CP-9020 less than $1 \mathrm{ppm}$, and increasedslowly as the dosage from $1 \mathrm{ppm}$ to $8 \mathrm{ppm}$ with the turbidity removal rateup to $98.6 \%$. The results of flocculation efficiency of AP-1000 and NP-800 show that theturbidity removal rate of ultra-fine particles suspension increases rapidly at the lower dosages of AP-1000 and NP-800 and decreases rapidly as the dosage increases to $5 \mathrm{ppm}$, with the optimal turbidity removal rates at $94.4 \%$ for AP-1000 at $1 \mathrm{ppm}$ and $90.9 \%$ for NP-800at 0.8 , respectively.

Comparison of the flocculation efficiency among three different types of polyacrylamide above, CP-9020 maintains a high removal rate in the dosage ranging from 3 to $10 \mathrm{ppm}$, which flocculation efficiency is obviously higher than those of AP-1000 and NP-800. This is likely because CP-9020 has cationic group which has a much stronger affinity toward the anionic ultrafine particles than anionic polyacrylamide AP-1000 which could cause electrostatic repulsion with ultra-fine particles. The molecular chains of the polyacrylamides are long enough to play a critical role in bridging fine particles. The dual role of bridging and charge neutralization is contribute to the effective flocculation of ultra-fine particles to form larger particles and settlement ${ }^{[5]}$. 


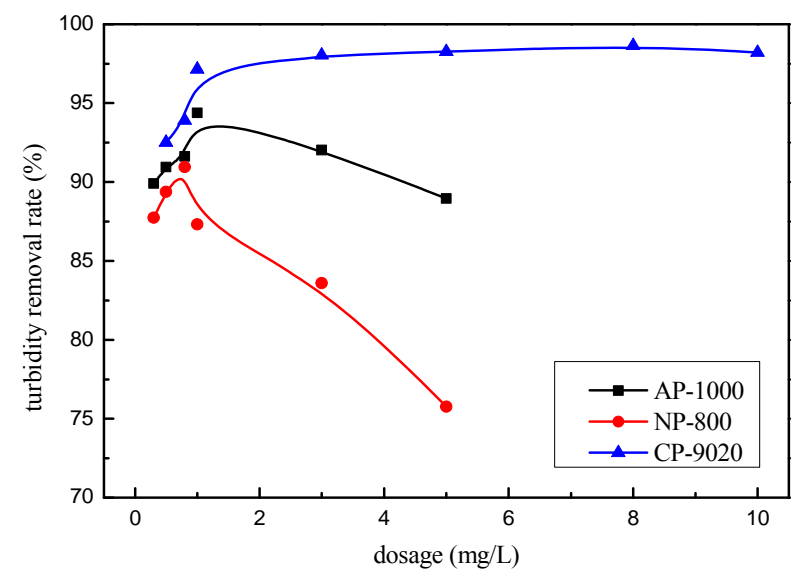

Fig.1 flocculation efficiency of different type of PAM on ultra-fine particles

\section{Effect of molecular weight}

The results of flocculation of cationic polyacrylamides Percol 164 and Percol 63 with similar charge density and different molecular weight are shownin Figure 2.It has been found thattheturbidity removal rate of ultra-fine particles suspension increases rapidly and then decreases as the dosage of Percol 164 and Percol 63 increases. At the optimal dosage of 3 ppmfor both cationic polyacrylamides, the optimal turbidity removal rate of suspension reached to $99.1 \%$ by Percol 164 and $98.1 \%$ by Percol 63. CPAMwith a higher molecular weight behaved better in flocculation. Polymeric flocculants with long chain stretching in solution could readily adsorb on the surface of one or more particles, bridging several particles to form large flocs. The highermolecular weight means longer polymer chains and more adsorption vacancy for the chance of collision between particles and polymer chains, which contribute to the adsorption and bridging action.

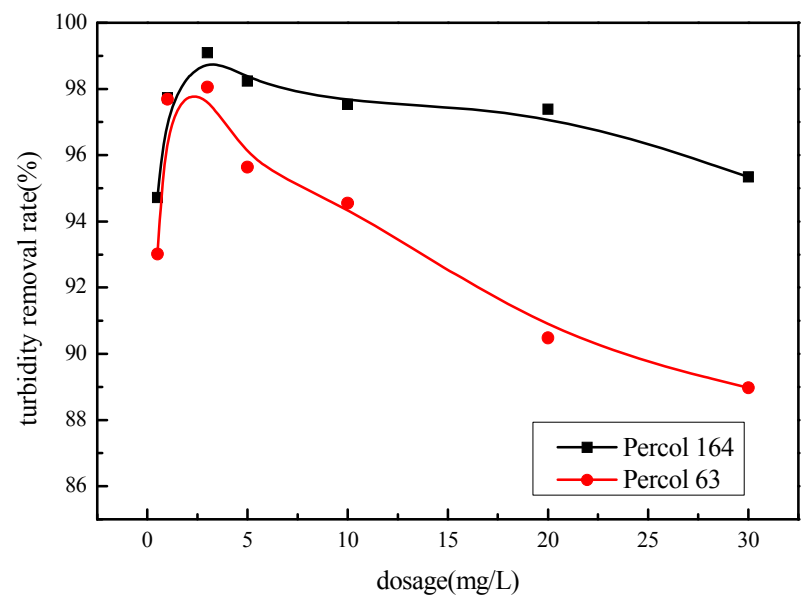

Fig.2 flocculation efficiency of different molecular weights of CPAM on ultra-fine particles

\section{Effect of charge density}

Figure 3 displays the results of flocculation efficiency of cationic polyacrylamides Percol 292 and Percol 63 with similar molecular weight but different charge density. It has been obviously found that the turbidity removal rate of ultra-fine particles suspension increases rapidly and then decreases as the dosages of Percol 292 and Percol 63 increase. The optimal turbidity removal rate of suspension flocculated by Percol 292 is $97 \%$ at 5 ppm, and by Percol 63 is $98.1 \%$ at 3 ppm, which indicates that Percol 63 performs better flocculation than Percol 292 even at a lower dosage. It might be because Percol 63 with higher charge density has more cationic groups, which provide more active sites and higher electrophoretic mobility for a given number of ultra-fine particles to generate electric neutralization action. 
The results on size meansurements indicated that the size of unflocculated ultra-fine particles ranged from $0.46 \mu \mathrm{m}$ to $11.2 \mu \mathrm{m}$ and the size of ultra-fine particles agglomerated was larger obviously. In comparison with the average particle size, the unflocculated ultra-fine particles is $2.57 \mu \mathrm{m}$; whereas the flocculated ones have average sizes from $98 \mu \mathrm{m}$ up to 200 , demonstrating the success of agglomeration. Especially, the average particle size of ultra-fine particles flocculated by CP-9020 was about 80 times higher than that before the flocculation.

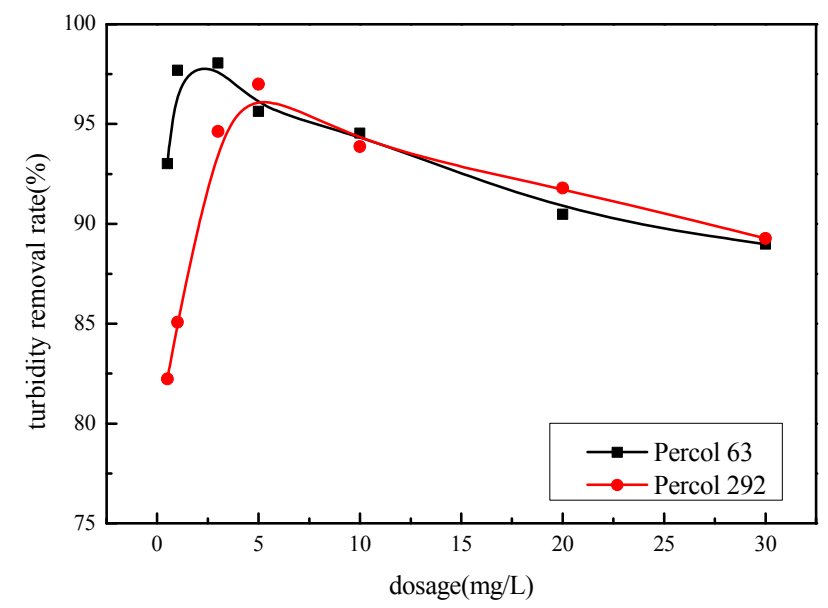

Fig.3 flocculation efficiency of different charge density of CPAM on ultra-fine particles

\section{CONCLUSIONS}

The main conclusions from this work are:

(1) Polyacrylamides with different type, molecular weight and charge density behaved different flocculation effects on ultra-fine particles. The cationic polymer with high molecular weight and high charge density incudeed to agglomeration or flocculation effectively at relatively dosage (3 ppm

(2) Ultra-fine particles can be flocculated to larger sizes in liquid by polyacrylamides, especially by CP-9020 at the dosage of 8 ppm, reaching the average size about 80 times higher than that before the flocculation. The research of the ultra-fine particles flocculation in liquid will be instructive for the research of aggregation of the ultra-fine particles from flue gas of coal-fired power plant.

\section{ACKNOWLEGEMENTS}

This work was financially supported by Beijing science and technology innovation base cultivation and development of special projects (Z151100001615048), NSF China (No. 21466005 and 51379077) and Guangxi Natural Science Foundation (2014GXNSFAA118036).

\section{REFERENCES}

[1] Q. Yao,S.-Q. Li,H.-W. Xu,et al.Studies on formation and control of combustion particulate matter in China: A review[J]. Energy. 2009, 34: 1296-1309

[2] Ruud Meij, Henk te Winkel. The emissions and environmental impact of PM10 and trace elements from a modern coal-fired power plant equipped with ESP and wet FGD[J]. Fuel Processing Technology, 2004, 85:641-656

[3] Hao Chen, Zhongyang Luo, Jianping Jiang, et al. Effects of simultaneous acoustic and electric fields on removal of fine particles emitted fromcoal combustion[J]. Powder Technology. 2015, 281: 12-19

[4] Dong Zhou, Zhongyang Lu, Mengxiang Fang, et al. Preliminary experimental study of acoustic agglomeration of coal-fired fine particles[J]. Procedia Engineering.2015,102: 1261-1270 
[5] Cherie Ovenden, Huining Xiao.Flocculation behaviour and mechanisms of cationic inorganic microparticle/polymer systems[J]. Colloids and Surfaces A: Physicochemical and Engineering Aspects. 2002,197: 225-234 